\title{
Aeromonas caviae mimicking Vibrio cholerae infectious enteropathy in a cholera-endemic region with possible public health consequences: two case reports
}

Marco van Zwetselaar ${ }^{1}$, Balthazar Nyombi ${ }^{2,3}$, Tolbert Sonda ${ }^{1,3}$, Happiness Kumburu ${ }^{1,3}$, Nyasatu Chamba ${ }^{2}$, Marieke C. J. Dekker ${ }^{2}$, Kajiru G. Kilonzo ${ }^{2,3}$, Sarah J. Urasa ${ }^{2,3}$ and Blandina T. Mmbaga ${ }^{1,2,3^{*}}$ (D)

\begin{abstract}
Background: Aeromonas species have been documented to yield false positive results in microbiological tests for Vibrio cholerae. They share many biochemical properties with Vibrio species, with which they were jointly classified in the family Vibrionaceae until genotypic information provided new insights. Aeromonas species are increasingly associated with gastrointestinal infections, albeit with great apparent variation in pathogenicity and virulence both between and within species of the genus. We report two cases with clinically mild cholera-like symptoms, at a time when a cholera outbreak was unfolding in other regions of the country (Tanzania). These are the first cases to be reported with Aeromonas mimicking cholera in our area.
\end{abstract}

Case presentation: Two patients were admitted at the isolation unit designated by the Kilimanjaro Christian Medical Centre for emerging infectious diseases and provided informed consent about regular stool analysis and culture under the provisional diagnosis of gastroenteritis. The first patient was a 23-year-old black African woman with a 2-day history of watery diarrhea and vomiting associated with a temperature of $39.7^{\circ} \mathrm{C}$. The second patient was a 47-year-old black African woman with a 2-day history of diarrhea and vomiting with a temperature of $37.7^{\circ} \mathrm{C}$, and she was hemodynamically stable. Both patients were isolated in a specific area for infection control and treated with fluids and orally administered rehydration solution, ciprofloxacin, metronidazole, and paracetamol. Stool culture was done. The isolated colonies were reported as $V$. cholerae and transferred to the research laboratory of Kilimanjaro Clinical Research Institute for confirmation using whole genome sequencing. Microbiological testing determined colonies isolated from stool to be V. cholerae, and warranted the conclusion "presumptive cholera." Whole genome sequencing, however, established the presence of Aeromonas caviae rather than $V$. cholerae.

Conclusions: The co-existence of Aeromonas species with $V$. cholerae in cholera-endemic regions suggests the possibility that a proportion of suspected cholera cases may be Aeromonas infections. However, with close to no epidemiological data available on Aeromonas infection in cases of diarrhea and dysentery in Sub-Saharan Africa, it is not currently possible to establish the extent of misdiagnosis to any degree of certainty. Whole genome sequencing was shown to readily exclude $V$. cholerae as the etiological agent and establish the presence of Aeromonas species.

Keywords: Diarrhea, Cholera outbreak, Aeromonas spp., V. cholerae, Whole genome sequencing

\footnotetext{
* Correspondence: b.mmbaga@kcri.ac.tz

${ }^{1}$ Kilimanjaro Clinical Research Institute, Moshi, Kilimanjaro, United Republic of

Tanzania

${ }^{2}$ Kilimanjaro Christian Medical Centre, Moshi, Kilimanjaro, United Republic of

Tanzania

Full list of author information is available at the end of the article
} 


\section{Background}

Detection of Aeromonas species, and in particular differentiating these from Vibrio species, can require a large number of laboratory tests [1-3]. We were caught out by this when on the same day two patients presented with cholera-like symptoms at a time when a major cholera outbreak was unfolding in several other regions in the country (Tanzania). We present this case report as a cautionary tale, with possible relevance for the management of cholera outbreaks. Having obtained the whole genome sequences of the isolates we also hope to make a modest contribution to the growing evidence linking Aeromonas with human enteropathy.

Tanzania experienced two large cholera outbreaks in 2015. In a refugee camp on the Tanzania-Burundi border, 4833 cases had been reported, of which 40 died [4]. A new index case presented in Dar es Salaam on 15 August 2015. Our cases presented on 8 September 2015, when 857 cases and 13 deaths had been reported countrywide. By October, the outbreak had spread to 13 regions, with 4835 cumulative cases $[5,6]$. When the outbreak abated in April 2016, a total of 24,018 cases had been reported in 28 regions, of which 429 died [7].

Cholera is an acute diarrheal infection caused by ingestion of food or water contaminated with the bacterium Vibrio cholerae. Cholera is a potentially fatal disease affecting adults and children, and can kill within hours if untreated. The $\mathrm{O} 1$ and $\mathrm{O} 139$ serogroups of $V$. cholerae cause outbreaks; other strains can cause mild diarrhea but do not generate epidemics. Approximately $80 \%$ of people infected with $V$. cholerae do not develop symptoms; they can, however, shed the bacteria for up to 10 days. Among those who develop symptoms, $80 \%$ have mild or moderate symptoms, while around $20 \%$ develop acute watery diarrhea with severe dehydration [8].

Aeromonas species have been associated with a wide variety of human infectious diseases including gastroenteritis, wound infections, septicemia, respiratory infections, hepatobiliary infections, and urinary tract infections [3, 9]. Aeromonas is most commonly associated with gastrointestinal infections. Most Aeromonas-associated enteropathy is watery diarrhea with a self-limited course; however, fever, abdominal pain, vomiting, bloody diarrhea, and secondary dehydration may occur. Outbreaks of Aeromonas-associated enteropathy have rarely been reported. In one outbreak of diarrhea with 2170 cases, Aeromonas was the most prevalently isolated single enteric pathogen (82\%), but $V$. cholerae strain $\mathrm{O} 1$ was also isolated in $12 \%$ of samples, and identified as the more likely etiological agent [10].

Aeromonas is ubiquitous in aquatic environments. Vibrio and Aeromonas have been shown to co-inhabit a shared host. The non-biting midges (chironomids), an abundant insect in aquatic habitats [11], have been proposed as a natural reservoir for Vibrio and Aeromonas species [12].
Very little is known about the incidence of Aeromonasassociated gastroenteritis in Sub-Saharan Africa (SSA). Studies in a number of Asian, Latin-American, and African countries isolated Aeromonas in 1 to $80 \%$ of children with diarrhea, and in 0 to $45 \%$ of asymptomatic children [13]. The recent large-scale Global Enteric Multicenter Study (GEMS) study [14] found Aeromonas infection to be significantly associated with watery diarrhea and dysentery in children under 5 in Bangladesh and Pakistan, but found no significant attributable risk for the sites in India or the four SSA countries in the study. In Bangladesh and Pakistan, the study found Aeromonas in 18.7 to $28.6 \%$ of stools of children with medium to severe diarrhea, usually with co-infecting enteropathogens. It was found as the sole pathogen in only 1.8 to $4.2 \%$ of cases. Aeromonas was detected in the stools of approximately equal numbers of cases and matched controls [14-16].

The epidemiology of Aeromonas-associated diarrhea is influenced by host and environmental factors, and by variation in pathogenicity between strains $[17,18]$. Isolates were shown to exhibit large variation in the presence of virulence genes, both between and within species $[17,19,20]$.

Up to the 1980s, the genera Aeromonas and Vibrio were classified jointly in the family Vibrionaceae. Molecular genetic developments then entailed reclassification into its proper family of Aeromonadaceae. Several reclassifications within the genus have since taken place. At least 31 acknowledged or proposed species of Aeromonas have been described, exhibiting great phenetic diversity [17]. The dominant species associated with human enteropathology are Aeromonas caviae, Aeromonas hydrophilae, and Aeromonas veronii biovar sobria, although studies disagree on the relative importance of these species in human infections [13, 21-24].

To distinguish Aeromonas species from their former family members $V$. cholerae and Plesiomonas shigelloides (all oxidase positive, setting them apart from the Enterobacteriaceae), the key screening reactions include: growth in nutrient broth in the presence and absence of salt; growth on thiosulfate-citrate-bile salts-sucrose (TCBS) agar (selective for $V$. cholerae with circular yellow or green $>2 \mathrm{~mm}$ colonies, inhibitive to Aeromonas with no or tiny colonies); resistance to vibriostatic agent $\mathrm{O} / 129$; and the string test [3]. Ghenghesh et al. (2008) documented laboratory procedures requiring minimal resources, enabling application in low-resource settings [13]. Aeromonas strains have been documented to exhibit atypical results for various assays and can in rare cases "be mistakenly identified as $V$. cholerae if a complete battery of screening tests aren't performed" [1]. The UK's Public Health England standard for identification of Vibrio species was expanded in 2015 to include procedures for differentiation of Aeromonas species [25]. 
Genomic data will readily and accurately identify isolates as members of the Aeromonas genus, although typing to species rank is complicated by multiple factors. Classification by comparison with publicly deposited genomes, even in curated databases, can result in mislabeling [22]. This is in part due to posterior reassignments within the genus, but also inherent to Aeromonas biology. Recombination occurs frequently between members of the genus, creating "highways of gene transfer" that obscure vertical phylogeny [26]. In addition, the ribosomal RNA (rRNA) genes of Aeromonas species are characterized by an exceptionally high copy number and large intragenomic heterogeneity, defying the common identification measure of 16S rRNA gene identity [27-29].

In light of the intricate taxonomy of Aeromonas, whole genome content comparisons, as measured by in silico DNA-DNA hybridization and average nucleotide identities (ANI), have been proposed as the "new gold standard" for identification [22, 26]. In silico multi-locus sequence typing (MLST) using the conserved housekeeping genes $\operatorname{gyr} B, \operatorname{rpoD}, \operatorname{rec} A, \operatorname{dnaJ}, \operatorname{gyr} A$, and $d n a X$ was demonstrated to accurately reproduce the taxonomy of Aeromonas species [30].

For clinical purposes, detailed identification is arguably less relevant than determining the virulence and drug susceptibility profile of the organism, in particular when these characteristics do not segregate cleanly with species. Whole genome sequencing (WGS) has the benefit of providing data to analyze both, to the extent that mechanisms have been genotyped. WGS can additionally provide unprecedented insight into the dynamics of disease outbreaks.

With outbreaks recurring almost every year, cholera is an endemic disease in Tanzania, as in many other SSA countries. Its etiological agent, $V$. cholerae, co-exists with species of the phenotypically similar but genotypically distinct genus Aeromonas in the same region. Clinical cases of mild to severe infectious gastroenteritis may be caused by virulent strains of Aeromonas, although very little is known about its incidence in SSA. Laboratory tests for $V$. cholerae may give false positive results when assayed on Aeromonas species. Therefore, advanced testing - which is often unavailable in low-resource settings - can be required to establish Aeromonas infection, as this case report intends to illustrate.

\section{Case presentation}

Two patients were admitted at the isolation unit designated by the hospital for an emerging infectious diseases outbreak in one of the referral hospitals in Moshi, Tanzania, which is a tertiary referral hospital and academic training center. The two patients provided informed consent about regular stool testing with the indication of infectious gastroenteritis, as well as for analysis of the samples in further genomic studies.
The first patient was a 23-year-old black African woman with a 2-day history of diarrhea and vomiting. The day prior to admission, she had eaten a fried meat snack from a restaurant. Later in the night she had more than ten bouts of profuse yellowish-greenish and watery diarrhea, with colicky abdominal pains, vomiting only once. On examination, she was sick-looking but alert, not dehydrated, hemodynamically stable, and had $39.7^{\circ} \mathrm{C}$ fever.

The second patient was a 47-year-old black African woman with a 2-day history of diarrhea and vomiting. She came straight from her home, and had eaten her normal meal of meat, ugali (a starch provider), and vegetables, preparing the food as she always did. She had over ten bouts of profuse watery diarrhea, greenish-yellowish in color and associated with generalized abdominal pains that were colicky in nature, and vomited five times. On examination, she was sick-looking, alert, and not dehydrated with a temperature of $37.7{ }^{\circ} \mathrm{C}$, and she was hemodynamically stable. Both patients were isolated in a specific area for infection control and treated with fluids and orally administered rehydration solution, ciprofloxacin, metronidazole, and paracetamol.

\section{Microbiology}

In both patients, stool analysis and culture were done under the provisional diagnosis of gastroenteritis. The stool samples from the two patients at the Kilimanjaro Christian Medical Centre (KCMC) were subjected to a number of standard assays routinely performed in the case of suspected infectious gastroenteritis at the KCMC Clinical Laboratory. Stool microscopy assessment was done on Gram staining and wet preparation for motility. Rectal swabs were put in alkaline peptone water (APW; Sigma; Steinheim, Germany) for enrichment and incubated at $37{ }^{\circ} \mathrm{C}$ for 18 hours. The enriched samples were then subcultured onto TCBS agar (Sharlau, Spain). After 24 hours of incubation at $37^{\circ} \mathrm{C}$, yellow colonies (sucrose fermenting, 2-3 $\mathrm{mm}$ diameter) suspected as Vibrio cholera were characterized by Gram staining (Gram-negative comma-shaped rods), and biochemical testing was done for positive oxidase reaction, urea hydrolysis, citrate fermentation, sulfur reduction, and indole production in sulfide indole motility (SIM) medium. Serological typing by agglutination test with the polyvalent $\mathrm{O} 1$ antiserum was done on one isolate from case 2. Inaba and Ogawa sero-agglutination was not performed. Results are summarized in Table 1.

The isolated colonies were reported as presumptive $V$. cholerae, but the inconclusive evidence and discordance with clinical presentation necessitated confirmatory tests. Medication was changed to doxycycline for another 3 days. The patients progressed well, and stool samples were taken again for follow-up analysis. A full blood picture was essentially normal in both patients. 
Table 1 Microbiology results

\begin{tabular}{|c|c|c|c|c|c|c|c|c|}
\hline Test: & $\mathrm{KIA}$ & Urea & Citrate & SIM & Gas & Oxidase & Poly O1 & Inaba / Ogawa \\
\hline Case 1 & Acid / Acid & Neg & Neg & Neg / Neg / Pos & Neg & Pos & ND & $\mathrm{ND}$ \\
\hline Case 2 & Acid / Acid & Neg & Neg & Neg / Neg / Pos & $\mathrm{Neg}$ & Pos & Pos & ND \\
\hline
\end{tabular}

KIA Kligler Iron Agar, ND not done, Neg negative, Pos positive, SIM sulfite indole motility

\section{Genomics}

The isolates were transferred to the research laboratory of Kilimanjaro Clinical Research Institute (KCRI) for confirmation using WGS. KCRI is the research institute liaised to the referral facility and hosts one of the very few next generation sequencers in East Africa. Here the isolates were subcultured onto TCBS agar plates and incubated at $37{ }^{\circ} \mathrm{C}$ for 24 hours. Colonies from TCBS were used for Genomic DNA extraction. Genomic DNA was extracted using MasterPure ${ }^{\mathrm{Tm}}$ Complete DNA and RNA Purification Kit (Epicentre, Illumina Inc.). The quality and quantity of genomic DNA were accessed using Qubit 2.0 fluorometer (Invitrogen, Life Technologies). DNA library preparation (dual indexing) was done using Nextera XT DNA preparation kit (Illumina Inc.). WGS of the library was done on Illumina MiSeq (Illumina Inc., San Diego, USA) using 2 by 250 bp paired end protocol.

Initial genomic analysis of the sequencer reads was performed using the standard pipeline for WGS research at KCRI. The pipeline is built from local instances of the free online services at the Centre for Genomic Epidemiology of Danish Technical University (https://cge.cbs.dtu.dk/ services/). The pipeline successively performed: identification of species from raw reads (KmerFinder 1.1); de novo genome assembly and quality assessment (SPAdes 3.5, Quast 2.3); 16S rRNA species typing (SpeciesFinder 1.2); multi-locus typing (MLST 1.8); and antimicrobial resistance prediction (ResFinder 1.3). Additional analyses were performed to disambiguate the pipeline output, and investigate virulence genes (BLAST 2.2).

The sequencer run produced a below average yield (average base coverage 10.4), and as a consequence de novo assembly resulted in fragmented genome assemblies. Both samples produced approximately 450 contigs, with largest contig $\sim 90 \mathrm{kbp}$, and N50 size $\sim 20 \mathrm{kbp}$. This affected the discriminatory power of downstream analyses and precluded an exhaustive phylogenetic analysis, but sufficed for the clinical purpose of species identification.

KmerFinder results conclusively excluded $V$. cholerae, and established the isolates as genus Aeromonas, most likely species $A$. hydrophila. SpeciesFinder failed to $16 \mathrm{~S}$ type the assemblies, probably as a consequence of Aeromonas' characteristically high $16 \mathrm{~S}$ rRNA gene copy number, exacerbated by the problems that long repeats pose for genome assembly. Typing was then performed against the hand-curated GreenGenes $16 \mathrm{~S}$ and SILVA SSU/LSU reference datasets. Neither provided a taxonomic assignment more specific than genus Aeromonas. Subsequent local alignment of the assemblies to the NCBI 16SMicrobial database, and manually collating the matching contigs, produced sequences which for both isolates matched A. caviae (GenBank sequence NR_104824.1).

Conclusive evidence for species $A$. caviae was obtained from matching the six housekeeping genes identified by Martinez-Murcia et al. [30] against reference sequences. Both isolates had $100 \%$ identity matches with $A$. caviae strains on the $\operatorname{gyr} B$ and $r e c A$ genes, $>99.6 \%$ on the $r p o D$ and gyrA genes, and $>98.5 \%$ on the dnaJ and dnaX genes. The two KCMC isolates matched different alleles for multiple genes, proving it unlikely that the infections originated from a single source.

ResFinder detected the blaMOX-4 (>95.2\% identity) and $a m p H$ genes ( $>82.4 \%$ identity) coding for beta-lactam resistance in both isolates. In one isolate it detected the sul1 and $d$ frA-15 genes with $100 \%$ identity, coding for sulfonamide and trimethoprim resistance respectively.

The fragmented assembly prevented an exhaustive search for proposed virulence genes. The alt gene for thermo-labile cytotonic enterotoxin was found integrally in both isolates. The act gene for cytotoxic enterotoxin was absent in both. For several other genes (ast, hlyA, aerA, aexT, ascV) matching partial fragments (350-700 $\mathrm{bp)}$ were found, providing insufficient support to establish their presence.

\section{Discussion}

We report concomitant outbreaks of genuine $V$. cholerae infection along with cases of a less infectious and less virulent clinical cholera-mimic caused by $A$. caviae in the United Republic of Tanzania.

The initial microbiological tests which showed positivity for $V$. cholerae were an obvious reason for concern. An outbreak of cholera type $\mathrm{O} 1$ - or in our case the spread of an ongoing outbreak to a new and populous region - has widespread consequences. Routine further testing by the clinical laboratory did not disprove the hypothesis that the pathogen was $V$. cholerae. The suspicion of an alternative explanation for the gastroenteritis was raised by the clinically mild phenotype in the two patients from our hospital. This underscores the importance of clinical and laboratory scrutiny, especially when typical cases have been reported from the same greater region.

Aeromonas species may yield false positive microbiological test results for $V$. cholerae. The extent to which misdiagnosis occurs is unknown. Provisionally confirmed cases in suspected outbreaks which are rapidly contained 
probably go unreported. Not all O1 infections are fulminant, and all non-O1 $V$. cholerae infections have a clinical presentation which is very similar to that of Aeromonas enteric infection. The fact that in a separate study (in progress) we isolated Aeromonas from 2 out of 15 stool samples from cases of the 2015 cholera outbreaks, suggests that there is some extent of co-occurrence, and possible confounding, of the two infections during O1 outbreaks.

WGS straightforwardly produced the data required for species and strain identification, at a comparable cost to the USD 85-90 for sero-agglutination tests [31]. However a number of conditions must be met before WGS can be used as the "new gold standard." Curated reference sequences with validated taxonomical assignment must be available, and there must be agreement on decision procedures for establishing species identity. The case of $A$. caviae also demonstrates that automated bioinformatics pipelines which do not take into account species-specific genotypic idiosyncrasies may fail to correctly identify species. Some Aeromonas species defy $16 \mathrm{~S}$ rRNA typing even under the assumed ideal conditions of WGS, and need bespoke analysis. More generally, in order that reliable conclusions can be drawn from the diagnostics they provide, bioinformatics instruments need validation and calibration in terms of sensitivity and specificity. This gap particularly needs closing when WGS moves from research-only to clinical applications.

\section{Strength and limitation}

The strength of our cases was the isolation of Aeromonas species supported by availability of the WGS at KCRI and avoidance of a misdiagnosis. A limitation might be that the cases were from an isolation unit where cholera-suspected cases were admitted; as they were the only two cases admitted to the unit during the cholera outbreak there might be a possibility of missing other cases of gastroenteritis due to Aeromonas species in other wards in the hospital.

\section{Conclusions}

This case report shows that suspected cases of $V$. cholerae infection may require extensive testing to exclude Aeromonas species, notably $A$. caviae. It highlights the need for laboratory guidelines such as the UK guidelines [25] which pay attention to the possible confusion, ideally adapted to low-resource settings, to be applied in suspected cases of $V$. cholerae. WGS was shown to readily exclude $V$. cholerae as the etiological agent and establish the presence of Aeromonas species. WGS however is not routinely available, certainly not in Africa, nor will it soon replace classical microbiology as the first-line diagnostic for cases of enteropathy.

The co-occurrence of $V$. cholerae and Aeromonas species in cholera-endemic regions suggests the possibility that a proportion of suspected cholera cases may instead exhibit Aeromonas-associated gastroenteritis. However, the dearth of epidemiological data on Aeromonas infection in cases of diarrhea and dysentery, and in particular its near complete lack for SSA, makes it impossible to establish the extent of misdiagnosis with any degree of certainty.

However, if the qualification "an emergent human pathogen," which is almost idiomatically used in the literature on Aeromonas species, is ever to be promoted to "an endemic human pathogen," then there is a strong need for both comprehensive epidemiological data and reliable means of diagnosis.

\section{Patient's perspective}

On discussion of the result with one of the patients and the publication plan, she said that it was good that she knew she had not had cholera because she had had feelings of shame and stigma. She said: "I was wondering as how can I alone get cholera while the same food I prepared myself my children ate but none of them had similar disease as me, now I am happy and will tell the family I had no cholera."

\section{Acknowledgements \\ The authors are grateful to Frank Aarestrup and team at Danish Technical University for their assistance and encouragement. \\ Funding \\ MvZ, TS, and HK are partially funded through DANIDA Fellowship Centre award DFC12-007DTU.}

\section{Availability of data and materials}

Not applicable.

\section{Authors' contributions}

BN reported the clinical laboratory methods and findings; HK reported the research laboratory methods and findings and performed the genome sequencing; NC, KK, SJU, and BTM reported the clinical presentation; MvZ and TS executed the bioinformatics and reported the genomic analyses; MD brought the authors together and made the initial draft; BTM and MvZ authored the manuscript. All authors read and approved the final manuscript.

Ethics approval and consent to participate

This case report was done under the Whole genomic sequencing diagnosis study which obtained ethical approval from the college ethics review committee of the KCMUCo and the National Institute of Medical Research in Tanzania and consent for publication was obtained from the patient.

Consent for publication

Written informed consent was obtained from the patients for publication of this case report and any accompanying images. A copy of the written consent is available for review by the Editor-in-Chief of this journal.

Competing interests

The authors declare that they have no competing interests.

\section{Publisher's Note}

Springer Nature remains neutral with regard to jurisdictional claims in published maps and institutional affiliations. 


\section{Author details}

Kilimanjaro Clinical Research Institute, Moshi, Kilimanjaro, United Republic of Tanzania. ${ }^{2}$ Kilimanjaro Christian Medical Centre, Moshi, Kilimanjaro, United Republic of Tanzania. ${ }^{3}$ Kilimanjaro Christian Medical University College, Moshi, United Republic of Tanzania.

Received: 1 August 2017 Accepted: 2 February 2018

Published online: 17 March 2018

\section{References}

1. Abbott SL, Seli LS, Catino M, Hartley MA, Janda JM. Misidentification of Unusual Aeromonas Species as Members of the Genus Vibrio: a Continuing Problem. J Clin Microbiol. 1998:36:1103-4.

2. Abbott SL, Cheung WKW, Janda JM. The Genus Aeromonas: Biochemical Characteristics, Atypical Reactions, and Phenotypic Identification Schemes. J Clin Microbiol. 2003:41:2348-57.

3. Janda JM, Abbott SL. The Genus Aeromonas: Taxonomy, Pathogenicity, and Infection. Clin Microbiol Rev. 2010;23:35-73.

4. WHO Regional Office for Africa. WHO notified of new cholera outbreaks in Tanzania https://www.reliefweb.int/report/united-republic-tanzania/whonotified-new-cholera-outbreaks-tanzania. Accessed 5 Oct 2016.

5. WHO. Cholera - United Republic of Tanzania; Disease Outbreak News 21 Oct 2015. WHO. 2015; published online Oct 21. http://www.who.int/csr/ don/21-october-2015-cholera/en/ Accessed 5 Oct 2016.

6. WHO. Cholera - United Republic of Tanzania; Disease Outbreak News 26 Nov 2015. WHO. 2015; published online Nov 26. http://www.who.int/csr/ don/26-november-2015-cholera-tanzania/en/ Accessed 5 Oct 2016.

7. WHO. Cholera - United Republic of Tanzania; Disease Outbreak News 22 April 2016. WHO. 2016; published online April 22. http://www.who.int/csr/ don/22-april-2016-cholera-tanzania/en/. Accessed 5 Oct 2016.

8. WHO. WHO Cholera Fact Sheet. WHO. 2015; published online July. http:// www.who.int/mediacentre/factsheets/fs107/en/. Accessed 5 Oct 2016.

9. Igbinosa $\mathrm{IH}$, Igumbor EU, Aghdasi F, Tom M, Okoh Al. Emerging Aeromonas Species Infections and Their Significance in Public Health. Sci World J. 2012; 2012:1-13.

10. Mendes-Marques CL, do Nascimento LM, Theophilo GND, et al. Molecular characterization of Aeromonas spp. and Vibrio cholerae $\mathrm{O} 1$ isolated during a diarrhea outbreak. Rev Inst Med Trop São Paulo. 2012;54:299-304.

11. Laviad S, Halpern M. Chironomids' Relationship with Aeromonas Species. Front Microbiol. 2016;7. https://doi.org/10.3389/fmicb.2016.00736.

12. Senderovich $Y$, Gershtein $Y$, Halewa E, Halpern M. Vibrio cholerae and Aeromonas: do they share a mutual host? ISME J. 2008;2:276-83.

13. Ghenghesh KS, Ahmed SF, El-Khalek RA, Al-Gendy A, Klena J. Aeromonasassociated infections in developing countries. J Infect Dev Ctries. 2008;2:81-98.

14. Kotloff KL, Nataro JP, Blackwelder WC, et al. Burden and aetiology of diarrhoeal disease in infants and young children in developing countries (the Global Enteric Multicenter Study, GEMS): a prospective, case-control study. Lancet. 2013:382:209-22.

15. Liu J, Platts-Mills JA, Juma J, et al. Use of quantitative molecular diagnostic methods to identify causes of diarrhoea in children: a reanalysis of the GEMS case-control study. Lancet Lond Engl. 2016;388:1291-301.

16. Qamar FN, Nisar MI, Quadri F, et al. Aeromonas-Associated Diarrhea in Children Under 5 Years: The GEMS Experience. Am J Trop Med Hyg. 2016; published online Aug 15. https://doi.org/10.4269/ajtmh.16-0321.

17. Chen P-L, Wu C-J, Tsai P-J, et al. Virulence Diversity among Bacteremic Aeromonas Isolates: Ex Vivo, Animal, and Clinical Evidences. PLoS One. 2014; 9. https://doi.org/10.1371/journal.pone.0111213.

18. Ghenghesh KS, Ahmed SF, Cappuccinelli P, Klena JD. Genospecies and virulence factors of Aeromonas species in different sources in a North African country. Libyan J Med. 2014;9. https://doi.org/10.3402/ljm.v9.25497.

19. Tomás JM. The Main Aeromonas Pathogenic Factors. ISRN Microbiol. 2012; 2012. https://doi.org/10.5402/2012/256261.

20. Abdullah Al, Hart CA, Winstanley C. Molecular characterization and distribution of virulence-associated genes amongst Aeromonas isolates from Libya. J Appl Microbiol. 2003;95:1001-7.

21. Heuzenroeder MW, Wong CY, Flower RL. Distribution of two hemolytic toxin genes in clinical and environmental isolates of Aeromonas spp.: correlation with virulence in a suckling mouse model. FEMS Microbiol Lett. 1999:174:131-6

22. Beaz-Hidalgo R, Hossain MJ, Liles MR, Figueras M-J. Strategies to Avoid Wrongly Labelled Genomes Using as Example the Detected Wrong
Taxonomic Affiliation for Aeromonas Genomes in the GenBank Database. PLoS One. 2015;10. https://doi.org/10.1371/journal.pone.0115813.

23. Batra $P$, Mathur $P$, Misra MC. Aeromonas spp.: An Emerging Nosocomial Pathogen. J Lab Physicians. 2016;8:1-4.

24. Chen P-L, Lamy B, Ko W-C. Aeromonas dhakensis, an Increasingly Recognized Human Pathogen. Front Microbiol 2016;7. https://doi.org/ 10.3389/fmicb.2016.00793.

25. Public Health England. UK Standards for Microbiology Investigations: Identification of Vibrio and Aeromonas species. Public Health England; 2015. https://www.gov.uk/government/publications/smi-id-19-identification-ofvibrio-species. Accessed 23 Nov 2015

26. Colston SM, Fullmer MS, Beka L, Lamy B, Gogarten JP, Graf J. Bioinformatic Genome Comparisons for Taxonomic and Phylogenetic Assignments Using Aeromonas as a Test Case. MBio. 2014;5. https://doi.org/10.1128/mBio.02136-14.

27. Janda JM, Abbott SL. 16 S rRNA Gene Sequencing for Bacterial Identification in the Diagnostic Laboratory: Pluses, Perils, and Pitfalls. J Clin Microbiol. 2007:45:2761-4.

28. Roger F, Lamy B, Jumas-Bilak E, Kodjo A, Marchandin H. Ribosomal MultiOperon Diversity: An Original Perspective on the Genus Aeromonas. PLoS One 2012;7. https://doi.org/10.1371/journal.pone.0046268.

29. Roger F, Marchandin H, Jumas-Bilak E, Kodjo A, Lamy B. Multilocus genetics to reconstruct aeromonad evolution. BMC Microbiol. 2012;12:62.

30. Martinez-Murcia AJ, Monera A, Saavedra MJ, et al. Multilocus phylogenetic analysis of the genus Aeromonas. Syst Appl Microbiol. 2011;34:189-99.

31. Keddy KH, Sooka A, Parsons MB, Njanpop-Lafourcade B-M, Fitchet K, Smith AM Diagnosis of Vibrio cholerae 01 Infection in Africa. J Infect Dis. 2013;208:S23-31.

\section{Submit your next manuscript to BioMed Central and we will help you at every step:}

- We accept pre-submission inquiries

- Our selector tool helps you to find the most relevant journal

- We provide round the clock customer support

- Convenient online submission

- Thorough peer review

- Inclusion in PubMed and all major indexing services

- Maximum visibility for your research

Submit your manuscript at www.biomedcentral.com/submit
) Biomed Central 